\title{
Adesão ao tratamento medicamentoso em idosos cardiopatas
}

\author{
Adherence to drug treatment in elderly with heart conditions
}

\section{Adherencia al tratamiento farmacológico en ancianos con enfermedad cardíaca}

\section{RESUMO}

Objetivo: Avaliar a adesão ao tratamento medicamentoso em idosos cardiopatas. Método: Pesquisa de campo, descritiva e quantitativa. Consentiram em participar da pesquisa 40 idosos atendidos em um Ambulatório de Cardiogeriatria. A coleta de dados ocorreu de agosto a novembro de 2018, com dados sociodemográficos e clínicos, Avaliação Multidimensional da Pessoa Idosa à avaliação da adesão ao tratamento pelo Brief Medication Questionnaire. Resultados: A idade média foi de 74+9,9 anos do sexo feminino em $57,7 \%$ dos idosos. Segundo o $B M Q, 90 \%$ dos idosos apresentaram algum tipo de barreira para a adesão ao tratamento medicamentoso; $66,7 \%$ apresentaram barreiras de 'Regime', 10,2\% de 'Crenças' e 89,5\% de 'Recordação'. Conclusão: Na avaliação da adesão ao tratamento, segundo o $\mathrm{BMQ}$, as barreiras mais frequentes foram as de 'Recordação', seguidas de 'Regime' e de 'Crenças'. A frequência dos idosos com barreira de 'Recordação' foi maior entre os que utilizavam cinco ou mais medicamentos diários.

Descritores: Idoso de 80 anos ou mais; Enfermagem; Adesão à medicação; Idoso fragilizado; Cardiopatias.

\begin{abstract}
Purpose: To evaluate adherence to a drug treatment in elderly patients with heart diseases. Method: This is a descriptive, quantitative field research. Forty elderly people assisted in a Cardiogeriatrics Clinic consented to participate in the research. Data collection took place from August to November 2018 and comprised sociodemographic and clinical data, Multidimensional Assessment of the Elderly, and the assessment of adherence to treatment by the Brief Medication Questionnaire. Results: The mean age was $74 \pm 9.9$ years and $57.7 \%$ of the elderly were women. According to the $B M Q, 90 \%$ of the elderly had some type of resistance to adhering to drug treatment; $66.7 \%$ were due to 'Regime', $10.2 \%$ due to 'Beliefs' and $89.5 \%$ due to 'Recollection'. Conclusion: In assessing adherence to treatment according to the $\mathrm{BMQ}$, the most frequent reasons against adherence were 'Recollection', followed by 'Regime' and 'Beliefs'. The frequency of elderly people in the "Recollection" group was higher among those who took five or more medications per day.

Descriptors: Aged 80+; Nursing; Medication Adherence; Frail Elderly; Heart Diseases.
\end{abstract}

\section{RESUMEN}

Objetivo: Evaluar la adherencia al tratamiento farmacológico en pacientes ancianos con cardiopatía. Método: Investigación de campo, descriptiva y cuantitativa. Cuarenta ancianos atendidos en una Clínica de Cardiogeriatría dieron su consentimiento para participar en la investigación. La recolección de datos se realizó de agosto a noviembre de 2018, con datos sociodemográficos y clínicos, Evaluación Multidimensional del Anciano y la evaluación de la adherencia al tratamiento mediante el Cuestionario Breve de Medicación. Resultados: La edad media fue de $74+9,9$ años y el 57,7\% de los ancianos eran mujeres. Según el $\mathrm{BMQ}$, el $90 \%$ de los ancianos tenía algún tipo de barrera para la adherencia al tratamiento farmacológico; El 66,7\% tenía barreras de "Régimen", el 10,2\% de "Creencias" y el 89,5\% de "Recuerdo". Conclusión: Al evaluar la adherencia al tratamiento según $\mathrm{BMQ}$, las barreras más frecuentes fueron las de 'Recuerdo', seguidas de 'Régimen' y 'Creencias'. La frecuencia de las personas mayores con una barrera de "recuperación" fue mayor entre los que usaban cinco o más medicamentos al día.

Descriptores: Anciano de 80 o Más Años; Enfermería; Cumplimiento de la Medicación; Anciano Frágil; Cardiopatías.
Larissa Corigliano Reis ${ }^{1}$

D0000-0001-5965-1236

Rosimeire Angela Queiroz Soares ${ }^{1}$ (D) 0000-0002-3752-6634

Ronaldo Fernandes Rosa ${ }^{1}$ (D) $\underline{0000-0001-8744-2861}$

Luciana Gonzaga dos Santos Cardoso $^{1}$

0000-0001-6917-4904 ${ }^{1}$ Faculdade de Ciências Médicas da Santa
Casa de São Paulo.

Autor correspondente: Luciana Gonzaga dos Santos Cardoso

E-mail:

luciana.cardoso@fcmsantacasasp.edu.br

\section{Como citar este artigo:}

Reis LC, Soares RAQ, Rosa RF, Cardoso LGS. Adesão ao tratamento medicamentoso e fragilidade em idosos cardiopatas. Revista de Enfermagem do Centro-Oeste Mineiro. 2021;11:e4130. [Access__]; Available in:_. DOI: http://doi.org/10.19175/recom.v11i0.4130 


\section{INTRODUÇÃO}

Há décadas o Brasil passa por um período de transição demográfica, marcada principalmente pela redução acentuada da natalidade, associada à queda de mortalidade, com o aumento progressivo de pessoas com idades mais elevadas intensificando o processo de envelhecimento populacional ${ }^{(1)}$.

No Brasil, o indivíduo com sessenta anos ou mais é considerado idoso ${ }^{(2)}$. O número de idosos, ao final de 2019, era de 34 milhões de pessoas, correspondendo a $16,2 \%$ da população do país ${ }^{(3)}$.

O envelhecimento é um processo dinâmico, progressivo e irreversível, que atinge todos os indivíduos. Porém varia, de acordo com diversos fatores e, entre eles, as doenças crônicas com um impacto importante, fazendo com que as alterações decorrentes do envelhecimento sejam mais gradativas para alguns e mais rápidas para outros, dependendo de aspectos associados, como estilo de vida, cultura ou condições socioeconômicas ${ }^{(4)}$.

Entre as doenças crônicas, as mais frequentes entre os idosos são as cardiopatias, como a hipertensão arterial, doença arterial coronariana, insuficiência cardíaca e arritmias. Outras doenças, como diabetes mellitus, doenças pulmonares, doença vascular periférica, doenças musculoesqueléticas, renais, hepáticas, neoplasias e neurológicas são comorbidades nessa população(5).

Com isso, o uso de medicamentos torna-se muito comum entre os idosos, pois, pelas próprias características do envelhecimento, também são mais vulneráveis aos efeitos adversos dos fármacos, sendo necessária a utilização de outros medicamentos para o controle dessas reações ${ }^{(6)}$.

Dessa forma, a avaliação da adesão à terapia medicamentosa tem sido alvo de vários estudos nacionais e internacionais dada a magnitude da problemática para a saúde do idoso ${ }^{(5,7-14)}$. A adesão pode estar relacionada a fatores biológicos, psíquicos e sociais, como o declínio gradativo de algumas funções cognitivas e físicas ${ }^{(4)}$, ou ainda relacionada com a compreensão de valores e crenças em relação à doença e ao tratamento ${ }^{(4,15-}$ 16).

Adesão ao tratamento medicamentoso pode ser considerada como a utilização de pelo menos $80 \%$ dos medicamentos prescritos, incluindo o horário, a dose e o tempo de duração do tratamento. Desse modo, o uso incorreto, irracional ou a não utilização representariam a não adesão ao tratamento medicamentoso ${ }^{(12)}$. Também pode ser considerado que o 'papel do paciente seria o de sujeito ativo, que participa e assume responsabilidade sobre seu tratamento'(10).

Uma das causas de falhas no uso correto das medicações, caracterizando a não adesão ao tratamento medicamentoso entre idosos, descrita na literatura, é a chamada polifarmácia, definida como o 'uso de cinco ou mais medicamentos' ${ }^{\prime(6)}$. É comum os idosos terem mais de duas receitas médicas e ainda utilizarem outros fármacos como automedicação $0^{(5-7,9-11,17-18)}$.

Além disso, dados demográficos e socioeconômicos, capacidade funcional, condição clínica do paciente, ocorrência de múltiplas comorbidades e esquemas medicamentosos mostraram ter grande impacto na adesão ao tratamento ${ }^{(4,11,13,19)}$

Neste sentido, a adesão ao tratamento farmacológico é uma questão complexa e envolve múltiplos fatores, portanto é preciso conhecer as convicções culturais e sociais do paciente sobre a utilização de fármaco e assegurar que o indivíduo tenha informações claras e suficientes para que ele queira aderir ao tratamento, a partir de uma decisão apoiada e dialogada com os profissionais ${ }^{(10)}$

Por outro lado, observa-se também que a não adesão ao tratamento constitui uma das principais causas de piora clínica, resultando em aumento de complicações clínicas, funcionais e hospitalizações ${ }^{(8)}$.

Considerando que as características do envelhecimento e o uso frequente de medicamentos, em idosos cardiopatas, podem ser fatores de risco para a não adesão ao tratamento ${ }^{(10,14)}$, torna-se relevante verificar quais aspectos influenciam a adesão ao tratamento nessa população, para que sejam desenvolvidas ações estratégicas específicas com a finalidade de melhor controle das doenças crônicas entre os idosos.

Sendo assim, este trabalho teve como objetivo avaliar a adesão ao tratamento medicamentoso em idosos cardiopatas.

\section{MÉTODOS}

Pesquisa de campo, descritiva e de abordagem quantitativa dos dados, aprovada pelo Comitê de Ética em Pesquisa (CAAE: 87690918.8.0000.5479). A amostra foi selecionada por conveniência. Foram incluídos 40 idosos, com 
idade maior ou igual a 60 anos, orientados no tempo e espaço e que responderam corretamente questões sobre idade, data de nascimento e local de residência, atendidos em um Ambulatório de Cardiogeriatria, que recebe portadores de qualquer doença cardíaca ou cardiovascular com necessidade de acompanhamento do médico cardiologista. A coleta de dados ocorreu, no período de agosto a novembro de 2018 e foi realizada por acadêmicas do curso de enfermagem treinadas para a abordagem do idoso e aplicação dos instrumentos de coleta selecionados para este estudo. Os idosos que compareceram ao ambulatório para a consulta médica foram abordados na sala de espera e convidados a participar do estudo por meio do Termo de Consentimento Livre e Esclarecido (TCLE). Foram aplicados três formulários por meio de entrevista. o primeiro contendo informações sociodemográficas e clínicas, elaborado pelos autores, contendo idade, sexo, estado civil, escolaridade, ocupação, presença de cuidador e comorbidades. O segundo instrumento aplicado foi a Avaliação Multidimensional da Pessoa Idosa (AMPI) ${ }^{(20)}$ : Instrumento da Secretaria Municipal de Saúde de São Paulo, contendo 17 domínios (idade, autopercepção da saúde, arranjo familiar, condições crônicas, medicamentos, internações, quedas, visão, audição, limitação física, cognição, humor, atividades básicas da vida diária, atividades instrumentais da vida diária, incontinência, perda de peso não intencional e condições bucais). Esses domínios abordam aspectos para a avaliação das condições de saúde com a finalidade de identificar necessidades de saúde dos idosos. A pontuação pode variar de zero a 21 pontos. De zero a cinco pontos, idoso saudável; de seis a 10 pontos, idoso pré-frágil; maior ou igual a 11 pontos, idoso frágil. Para a avaliação da adesão ao tratamento medicamentoso, foi utilizado um terceiro instrumento, o Brief Medication Questionnaire $(B M Q)^{(21)}$, que inclui questões sobre medicamentos utilizados na última semana e problemas relatados sobre o uso das medicações. A pontuação é realizada de acordo com os problemas referidos e classificados em três aspectos. 'Regime': falha em listar os medicamentos prescritos, interrupção da terapia, falha em dias ou doses, redução ou omissão de medicamentos, dose extra ou medicação além das prescritas, não saber responder a alguma pergunta, se recusar a responder a alguma questão; 'Crenças': relatar se a medicação funciona bem e nomear medicamentos que incomodam; e 'Recordação': esquema de múltiplas doses e muita dificuldade ou alguma dificuldade para abrir ou fechar a embalagem, ler o que está escrito na embalagem, lembrar de tomar os medicamentos, conseguir o medicamento e tomar tantos comprimidos ao mesmo tempo. Para os idosos sem receita médica, não foram coletados dados sobre Regime, porém foram coletadas respostas sobre as barreiras de crença e recordação, pois, como o instrumento pode ser interpretado por barreira, considerou-se relevante manter as demais. Para a avaliação da adesão ao tratamento, escores maiores ou igual a um, em cada um desses aspectos, identifica barreiras relacionadas ao tratamento medicamentoso. Após o preenchimento dos formulários, as informações foram inseridas em um banco de dados do programa Excel e analisadas pela estatística descritiva. Para as variáveis quantitativas, foi apresentada a variação e mediana, média e desviopadrão. Para as variáveis qualitativas, foi apresentada a frequência relativa (\%).

\section{RESULTADOS}

A amostra foi composta por 40 pacientes. A idade variou de 61 a 94 anos, com mediana de 75, média de $74 \pm 9,9$ anos.

A Tabela 1 apresenta 0 perfil sociodemográfico e clínico dos idosos.

Tabela 1 - Frequência relativa segundo variáveis sociodemográficas e clínicas. São Paulo, 2018

\begin{tabular}{lc}
\hline Variáveis & $\%$ \\
\hline Sexo Feminino & 57,5 \\
Estado civil & \\
$\quad$ Casado & 55,0 \\
$\quad$ Viúvo & 35,0 \\
$\quad$ Solteiro/Divorciado & 10,0 \\
Escolaridade & \\
Analfabeto & 20,0 \\
Educação Básica & 43,3 \\
Ensino Superior & 36,7 \\
Ocupação & \\
Aposentado & 67,5 \\
Do lar & 17,5 \\
Trabalho remunerado & 15,0 \\
Cuidador & 15,0 \\
Hipertensão & 95,0 \\
Diabetes & 30,0 \\
Infarto prévio & 22,5 \\
Marcapasso & 10,0
\end{tabular}

Fonte: Elaborada pelos autores. 
A pontuação da AMPI variou de dois a 16 pontos, com mediana de 9 e média de $8,6 \pm 3,9$ pontos. $30,0 \%$ dos idosos obtiveram pontuação de até cinco pontos, sendo categorizados como saudáveis; 32,5\% pontuação entre 6 e 10 pontos, pré-frágeis; e 37,5\% com 11 pontos ou mais, frágeis.

Na AMPI observou-se que o domínio com maior frequência foi o uso de cinco ou mais medicamentos diariamente. Outros domínios com frequência maior ou igual a 50\% foram: Três ou mais doenças crônicas (57,5\%); Autopercepção de saúde regular, ruim ou muito ruim (52,5\%); Idade de 75 a 89 anos, dificuldades para ouvir, prótese mal adaptada ou dificuldades para mastigar ou engolir (50,0\%).

A Tabela 2 mostra a distribuição dos idosos segundo a categorização de cada domínio avaliado.

Tabela 2 - Frequência dos idosos em categorias dos 17 domínios da AMPI. São Paulo, 2018. N=40

\begin{tabular}{|c|c|c|}
\hline Domínios & Categorias & $\%$ \\
\hline Medicamentos (diário) & Usa cinco ou mais medicamentos diariamente & 75,0 \\
\hline Condições crônicas & Possui três ou mais condições crônicas & 57,5 \\
\hline Autopercepção de saúde & A autopercepção de saúde é regular/ruim/muito ruim & 52,5 \\
\hline Idade & Tem idade de 75 a 89 anos & 50,0 \\
\hline Audição & Refere dificuldades para ouvir & 50,0 \\
\hline Condições bucais & $\begin{array}{l}\text { A prótese está mal adaptada ou tem dificuldades para mastigar ou engolir o } \\
\text { alimento }\end{array}$ & 50,0 \\
\hline Cognição & Refere esquecimentos & 47,5 \\
\hline Quedas & Teve uma ou mais quedas no último ano & 45,0 \\
\hline Incontinência & Relata perda urinária & 40,0 \\
\hline Visão & Relata dificuldades para enxergar & 37,5 \\
\hline Humor & Relata desânimo, tristeza no último mês & 37,5 \\
\hline Arranjo familiar & Mora sozinho & 32,5 \\
\hline Atividades instrumentais da vida diária & Precisa de ajuda para sair de casa e lidar com o dinheiro & 32,5 \\
\hline Perda de peso não intencional & Perda de peso não intencional & 32,5 \\
\hline Internações no último ano & Refere uma ou mais internações no último ano & 30,0 \\
\hline Limitações físicas & Relata limitações físicas & 27,5 \\
\hline Atividades básicas da vida diária & Necessita de ajuda para sair da cama, banho, vestir-se ou alimentar-se & 12,5 \\
\hline
\end{tabular}

Fonte: Elaborada pelos autores.

Quanto à adesão ao tratamento medicamentoso avaliada pelo $\mathrm{BMQ}, 12$ idosos responderam as questões referentes à barreira de 'Regime', 39 às questões referentes à barreira de 'Crenças' e 38 barreiras de 'Recordação'. Observou-se que do total $90 \%$ dos idosos obtiveram pontuação maior ou igual a um, ou seja, apresentaram algum tipo de barreira para a adesão ao tratamento medicamentoso.

Na barreira de 'Regime', a pontuação variou de zero a sete pontos, com mediana de 1 e média de 1,6+1,3 pontos. 66,7\% apresentaram pontuação maior ou igual a um, indicando, portanto barreira de adesão ao tratamento nesse quesito.
Para as barreiras de 'Crenças', a pontuação variou de zero a um ponto, com mediana de 0 e média de 0,1+0,2 pontos. 10,2\% apresentaram pontuação igual a um, indicando barreira de adesão nesse quesito.

Quanto às barreiras de 'Recordação', a pontuação variou de zero a dois pontos, com mediana de 1 e média de 1,3+0,6 pontos. Apresentaram pontuação maior ou igual a um $89,5 \%$, demonstrando barreira de adesão nesse quesito.

A frequência dos problemas relatados em cada aspecto nas barreiras de regime, crenças e recordação estão apresentados na Tabela 3. 
Tabela 3 - Frequência relativa de idosos com barreiras de regime, crenças e recordação, segundo o Brief Medication Questionnaire. São Paulo, 2018

\begin{tabular}{lc}
\hline Barreiras & $\%$ \\
\hline REGIME & 41,7 \\
Falhou em listar (espontaneamente) os medicamentos prescritos no relato inicial & 41,7 \\
Respondeu que 'não sabia' a alguma das perguntas & 25,0 \\
Relatou alguma falha de dias ou de doses & 25,0 \\
Reduziu ou omitiu doses de algum medicamento & 16,7 \\
Interrompeu a terapia pelo atraso na dispensação da medicação ou outro motivo & 8,3 \\
Tomou alguma dose extra ou medicação a mais do que o prescrito & 8,3 \\
Recusou-se a responder a alguma das questões & 7,7 \\
CRENÇAS & 2,6 \\
Nomeou as medicações que o incomodam & 80,0 \\
Relatou que alguma medicação 'não funciona bem' ou 'não sei' & 42,5 \\
RECORDAÇÃo & 44,7 \\
Recebe um esquema de múltiplas doses de medicamentos (2 ou mais vezes/dia)? & 13,2 \\
Relata 'muita dificuldade' ou 'alguma dificuldade' no uso do medicamento & 10,5 \\
Dificuldades relatadas & 8,1 \\
Ler o que está escrito na embalagem & 8,1 \\
Conseguir o medicamento &
\end{tabular}

Fonte: Elaborada pelos autores.

Para verificar as frequências dos idosos que possuem barreiras de adesão ao tratamento, segundo o tipo de barreira, considerando-se o número de medicamentos utilizados diariamente, conforme a categorização da AMPI, observa-se, na Tabela 4, que as barreiras de 'Regime' e 'Crenças' possuem a mesma frequência de idosos que utilizam de um a quatro medicamentos diários e os que utilizam cinco ou mais medicamentos. Porém, na barreira de 'Recordação', a frequência de idosos que utilizam cinco ou mais medicamentos diariamente é maior que a frequência dos que utilizam de um a quatro medicamentos.

Tabela 4 - Frequência relativa de idosos segundo número de medicamentos diários e tipo de barreira de adesão ao tratamento medicamentoso. São Paulo, 2018

\begin{tabular}{lccc}
\multirow{2}{*}{$\begin{array}{c}\text { Número de } \\
\text { medicamentos/dia }\end{array}$} & Regime & Crenças & Recordação \\
\cline { 2 - 4 } & & & \\
\hline 1 a 4 & 50,0 & 50,0 & 35,3 \\
5 ou mais & 50,0 & 50,0 & 64,7 \\
\hline
\end{tabular}

Fonte: Elaborada pelos autores.

\section{DISCUSSÃO}

A proposta do estudo foi avaliar a adesão ao tratamento medicamentoso de idosos atendidos no serviço. Participaram da pesquisa 40 pacientes, com idade média de 74+9,9 anos.

Predominou o sexo feminino, casados, aposentados e portadores de hipertensão arterial. A maioria dos idosos possuía três ou mais doenças crônicas. Esses dados corroboram com a literatura atual, ao evidenciar a prevalência da Hipertensão Arterial Sistêmica (HAS) como principal doença cardiovascular nos idosos, frequentemente associada a outras doenças crônicas ${ }^{(11,14,16,19)}$.

Além disso, estudos semelhantes com maior número de idosos corroboram com esses achados, em que a maioria da população também era do sexo feminino, com média de idade semelhante, com três ou mais comorbidades e uso de polifarmácia ${ }^{(5,6,10,22)}$.

Os domínios da AMPI com maior frequência foram: uso de múltiplos medicamentos, três ou mais condições crônicas e autopercepção de saúde regular/ruim/muito ruim. Outros domínios relevantes foram a idade entre 75 a 89 anos, dificuldades para ouvir e condições bucais com prótese mal adaptada ou disfagia. Tais resultados foram similares aos obtidos em outros estudos populacionais $^{(22-23)}$.

Nesse sentido, a avaliação multifuncional permite conhecer a realidade desses idosos com múltiplas comorbidades e esquemas terapêuticos complexos, declínio funcional e social, para orientar e intervir adequadamente ${ }^{(23)}$. Ressalta-se o papel educativo primordial do enfermeiro, fornecendo orientações precisas, para assegurar a adesão ao tratamento farmacológico e não farmacológico.

Estudos correlacionam avaliação negativa da saúde com maior índice de mortalidade em idosos. O projeto Saúde, Bem-estar e Envelhecimento (SABE) avaliou um grande número de idosos e 
obteve resultados similares, em que $67,8 \%$ dos idosos tinham avaliação negativa de sua saúde (regular, ruim ou muito ruim) e identificou que idosos com autopercepção negativa da saúde apresentaram maior declínio funcional e maior risco de mortalidade ${ }^{(24)}$.

Além disso, assinala-se que o declínio funcional e cognitivo influencia negativamente na adesão à terapia em idosos ${ }^{(4-5)}$.

Quanto à condição nutricional, nesta pesquisa, um número expressivo dos pacientes, $(32,5 \%)$ referiram perda de peso não intencional. Em pacientes cardiopatas, é um fator que deve chamar a atenção, considerando que a perda de peso pode relacionar-se com quadros avançados de insuficiência cardíaca ${ }^{(5,14)}$.

A Organização Mundial da Saúde (OMS) recomenda que a saúde da pessoa idosa não pode estar baseada apenas na ausência de doenças, mas deve ser considerada a partir da perspectiva funcional(25). Não obstante, os idosos desta pesquisa foram avaliados segundo os critérios da AMPI a qual possibilita analisar a capacidade funcional, cognitiva, afetiva, da comunicação, da mobilidade e do equilíbrio, eliminação, nutrição e recursos sociais e ambientais em que os idosos estão inseridos ${ }^{(26)}$.

O uso de cinco ou mais medicamentos diários foi um resultado compatível com o fato de que a maioria dos idosos eram portadores de três ou mais doenças crônicas, caracterizando a polifarmácia. É, por isso, um dos principais fatores que comprometem a adesão terapêutica especialmente em idosos. Somado a isso, o estudo identificou que barreiras de recordação foram fatores potenciais para a não adesão ao tratamento medicamentoso. Tais resultados foram similares ao encontrados em outras pesquisas ${ }^{(6,17)}$ e possibilitam planejar estratégias que visam melhorar a adesão terapêutica, já que a polifarmácia é uma realidade típica nessa faixa etária ${ }^{(6)}$.

Os resultados apontaram pouca influência da Barreira de crenças para a adesão, 10,2\% dos idosos queixaram-se de incômodo ou mau funcionamento da medicação.

Embora quase todos os pacientes reconheçam os benefícios do medicamento, relatando que 'funcionam bem', a maioria apresentou barreiras de 'Regime' e barreiras de 'Recordação'.

Nas Barreiras de recordação, a dificuldade mais frequente foi a dificuldade de ler o que está escrito na embalagem. Esse dado pode estar correlacionado a condições demográficas dos pacientes, em que uma porcentagem era de analfabetos, outros tinham dificuldades para enxergar e outros ainda moravam sozinhos. Estudos concluíram que o apoio familiar e o suporte social contribuem para a manutenção do tratamento, acompanhando ao serviço de saúde e auxiliando na tomada de medicamentos ${ }^{(27)}$.

A Tabela 4 também mostra que a frequência de idosos com barreira de recordação foi maior entre os que usam cinco ou mais medicamentos diários. Verifica-se que o uso da polifarmácia, geralmente associado à presença de múltiplas comorbidades, prejudica a adesão, uma vez que corrobora para a dificuldade de seguir o esquema terapêutico e para o esquecimento de tomar a medicação, tal qual demonstrado em outras pesquisas ${ }^{(11-12,14)}$.

Outras dificuldades no uso dos medicamentos foram: conseguir o medicamento, abrir ou fechar a embalagem, lembrar de tomar ou conseguir o medicamento e ingerir um número grande de comprimidos ao mesmo tempo. Esses dados são compatíveis com os obtidos em outros estudos sobre barreiras de adesão em idosos portadores de cardiopatias ${ }^{(5,13-14)}$.

Estudos demonstram que o conhecimento das principais dificuldades vivenciadas pelos idosos permitem reconhecer barreiras que dificultam a adesão ao tratamento, para estabelecer estratégias que minimizem as dificuldades, melhorando a adesão e, consequentemente, seu o estado de saúde ${ }^{(12,27)}$.

Pontua-se a importância de que a assistência seja realizada de forma integral e ao mesmo tempo individualizada, para conhecer as necessidades específicas dessa população, visando a estratégias que considerem e minimizem as dificuldades mais observadas entre os idosos ${ }^{(5)}$.

\section{CONCLUSÃO}

$\mathrm{Na}$ avaliação da adesão ao tratamento medicamentoso, segundo o $\mathrm{BMQ}$, as barreiras mais frequentes foram as de 'Recordação', seguidas das barreiras de 'Regime' e as barreiras de 'Crenças'. Nas barreiras de 'Regime', as respostas mais frequentes foram: Falhou em listar os medicamentos prescritos e Respondeu que 'não sabia' a alguma das perguntas. Nas barreiras de 'Crenças', a resposta mais frequente foi: Nomeiam as medicações que o incomodam e nas barreiras de 'Recordação', a resposta: Recebe esquema de múltiplas doses. A maior dificuldade relatada foi a 
de ler o que está escrito na embalagem. A frequência de idosos com barreiras de 'Recordação' foi maior entre os que utilizavam cinco ou mais medicamentos diários. Quanto às limitações do estudo, a pequena amostra e a impossibilidade de identificar as barreiras de regime, na maioria dos idosos, pela falta de receita médica para a conferência dos medicamentos prescritos. As dificuldades de adesão ao tratamento medicamento em idosos é um fato conhecido. Identificar os tipos de barreira para esta adesão contribui para que a assistência de enfermagem seja planejada e implementada a partir das necessidades mais comuns. Recomendase o desenvolvimento de novos estudos com amostras maiores, com possibilidade de estatística analítica para a identificação de fatores que influenciam a não adesão ao tratamento medicamentoso em idosos cardiopatas.

\section{REFERÊNCIAS}

1 - Oliveira AS. Transição demográfica, transição epidemiológica e envelhecimento populacional no Brasil. Hygeia 2019;15(32):69-79. DOI: 10.14393/Hygeia153248614

2 - Brasil. Ministério da Saúde. Estatuto do idoso. 3 ed. Brasília: Ministério da Saúde; 2013.

3 - Departamento Intersetorial de Estatística e Estudos Socioeconômicos. Quem são os idosos brasileiros. Bol Especial 2020 [citado em 15 mar 2021]; 1:1-3. Acesso em: https://www.dieese.org.br/boletimespecial/2020/ boletimEspecial01.html

4 - Castro-Costa E, Lima-Costa MF, Adrade FB, Souza Júnior PRB, Ferri CP. Função cognitiva entre adultos mais velhos: Resultados do ELSI-Brasil. Rev Saúde Pública 2018;52(supl 2):1s-9s. DOI: $\underline{10.11606 / s 1518-8787.2018052000629}$

5 - Aquino GA, Cruz DT, Silveiro MS, Vieira MT, Bastos RR, Leite ICG. Fatores associados à adesão ao tratamento farmacológico em idosos que utilizam medicamento anti hipertensivo. Rev Bras Geriatr Gerontol. 2017;20(1):116-27. DOI: $\underline{10.1590 / 1981-22562017020.160098}$

6 - Secoli SR. Polifarmácia: Interações e reações adversas no uso de medicamentos por idosos. Rev Bras Enferm. 2010;63(1):136-40. DOI: $\underline{10.1590 / 50034-71672010000100023}$
7 - Abreu DPG, Santos SSC, Ilha S, Silva BT, Martins NFF, Varela CS. Fatores comportamentais associados à adesão medicamentosa em idosos em atendimento ambulatorial. Rev Enferm Cent-Oeste Min. 2019;9:e3025. DOI: 10.19175/recom.v9i0.3025

8 - Corrêa NB, Faria AP, Moreno Júnior H, Modolo R. Não adesão ao tratamento farmacológico antihipertensivo como causa de controle inadequado da hipertensão arterial. Rev Bras Hipertens. 2016 [citado em 15 mar 2021]; 23(3):58-65. Acesso em: https://docs.bvsalud.org/biblioref/2018/03/88024 2/rbh-v23n3 58-65.pdf

9 - Maciel FM, Carneiro LBP, Real APB, Catro BCR, Amorim FM, Lopes GJ, et al. Frequência de baixa adesão e fatores relacionados em idosos atendidos em Ponto dos Volantes, Vale do Jequitinhonha. Geriatr Gerontol Aging. 2019;13(1):11-6. DOI: 10.5327/Z2447-211520191800064

10 - Freitas JGA, Nielson SEO, Porto CC. Adesão ao tratamento farmacológico em idosos hipertensos: Uma revisão integrativa da literatura. Rev Soc Bras Clín Méd. 2015 [citado em 15 mar 2021]; 13(1):75$84 . \quad$ Acesso em: http://www.sbcm.org.br/ojs3/index.php/rsbcm/ar ticle/view/122/118

11 - Tavares NU, Bertoldi AD. Fatores associados à baixa adesão ao tratamento medicamentoso em idosos. Rev Saúde Pública 2013;47(6):1092-101. DOI: $10.1590 / 50034-8910.2013047004834$

12 - Arruda DCJ, Eto FN, Velten APC, Morelato RL, Oliveira ERA. Fatores associados a não adesão medicamentosa entre os idosos de um ambulatório filantrópico do Espírito Santo. Rev Bras Geriatr Gerontol. 2015;18(2):327-37. DOI: 10.1590/1809-9823.2015.14074

13 - Raymundo CAN, Pierin AMG. Adesão ao tratamento de hipertensos em um programa de gestão de doenças crônicas: Estudo longitudinal retrospectivo. Rev Esc Enferm USP 2014;48(5):8119. DOI: $10.1590 / 50080-6234201400005000006$

14 - Resende AKM, Lira JAC, Prudêncio FA, Sousa LS, Brito JFP, Ribeiro JF, et al. Dificuldades de idosos na adesão ao tratamento da hipertensão arterial. Rev Enferm UFPE 2018;12(10):2546-54. DOI: 10.5205/1981-8963-v12i10a236078p2546-2554$\underline{2018}$ 
15 - Almeida GNA, Souza MAN, Lima-Costa MF, Peixoto S. Lifestyle factors and multimorbidity among older adults (ELSI-Brazil). Eur J Ageing 2020;17:521-9. DOI: 10.1007/s10433-020-00560$\underline{z}$

16 - Bonfim RA, Schneider IJC, Andrade FB, LimaCosta MF, Corrêa VP, Frazão $P$, et al. Racial inequities in tooth loss among older Brazilian adults: A decomposition analysis. Community Dent Oral Epidemiol. 2020;49(2):119-27. DOI: $\underline{10.1111 / \text { cdoe. } 12583}$

17 - Medeiros-Souza P, Santos-Neto LL, Kusano LTE, Pereira MG. Diagnosis and control of polypharmacy in the elderly. Rev Saúde Pública 2007;41(6):1049-5. DOI: 10.1590/50034$\underline{89102006005000050}$

18 - Andrade JM, Duarte YA, Alves LC, Andrade FCD, Souza Júnior PR, Lima-Costa MF, et al. Fraity profile in Brazilian older adults. ELSI-Brazil. Rev Saúde Pública 2018;52(supl 2):1s-17s. DOI: 10.11606/s1518-8787.2018052000616

19 - Pimenta FB, Pinho L, Silveira MF, Botelho ACC. Fatores associados a doenças crônicas em idosos atendidos pela Estratégia de Saúde da Família. Cienc Saúde Coletiva 2015;20(8):2489-98. DOI: 10.1590/1413-81232015208.11742014

20 - Prefeitura Municipal de São Paulo. Secretaria Municipal da Saúde. Documento norteador: Programa Acompanhante de Idosos. São Paulo: SMS; 2016.

21 - Ben AJ, Neumann CR, Mengue SS. Teste de Morisky-Green e Brief Medication Questionnaire para avaliar adesão a medicamentos. Rev Saúde Pública 2012;46(2):279-89. DOI: 10.1590/S003489102012005000013

22 - Diniz MA, Tavares DMS. Fatores de risco para doenças cardiovasculares em idosos de um município do interior de Minas Gerais. Texto Contexto-Enferm. 2013;22(4):885-92. DOI: 10.1590/S0104-07072013000400003

23 - Tavares DMS, Guimarães MO, Ferreira PCS, Dias FA, Martins NPF, Rodrigues LR. Qualidade de vida e adesão ao tratamento farmacológico entre idosos hipertensos. Rev Bras Enferm. 2016;69(1):134-41. DOI: 10.1590/0034$\underline{7167.2016690118 \mathrm{i}}$
24 - Lebrão ML, Duarte YAO. O Projeto SABE no Município de São Paulo: Uma abordagem inicial. Brasília: OPAS; 2003.

25 - World Health Organization (WHO). World Report on Ageing and Heath. Geneva: WHO; 2015.

26 - Fabricio SCC, Rodrigues RAP. Revisão da literatura sobre fragilidade e sua relação com o envelhecimento. Rev Rene 2008 [citado em 15 mar 2021]; 9(2):113-9. Acesso em: http://www.periodicos.ufc.br/rene/article/view/5 $\underline{046 / 3706}$

27 - Giacomin KC, Duarte YA, Camarano AA, Nunes DP, Fernandes D. Care and functional disabilities in daily activities- ELSI-Brazil. Rev Saúde Pública 2018;52(supl 2):1s-9s. DOI: 10.11606/s15188787.2018052000650

Nota: Esta pesquisa recebeu apoio do Programa Institucional de Bolsas de Iniciação Científica do Conselho Nacional de Desenvolvimento Científico e Tecnológico - PIBIC-CNPq e do Fundo de Amparo à Pesquisa da Faculdade de Ciências Médicas da Santa Casa de São Paulo.

Recebido em: 02/12/2020

Aprovado em: 28/04/2021 